2010-01-01

\title{
Ultrasound-Assisted SWNTs Dispersion: Effects of Sonication Parameters and Solvent Properties
}

\author{
Qiaohuan Cheng \\ Technological University Dublin, qiaohuan.cheng@tudublin.ie \\ Sourabhi Debnath \\ Technological University Dublin, Sourabhi.debnath@tudublin.ie \\ Elizabeth Gregan \\ Technological University Dublin, Elizabeth.Gregan@tudublin.ie
}

See next page for additional authors

Follow this and additional works at: https://arrow.tudublin.ie/nanolart

Part of the Nanoscience and Nanotechnology Commons

\section{Recommended Citation}

Cheng, Q. et al (2010) Ultrasound-Assisted SWNTs Dispersion:Effects of Sonication Parameters and Solvent Properties. Journal of Physical Chemistry C., Vol.114, (19), pp. 8821-8827. doi:10.1021/ jp101431h

This Article is brought to you for free and open access by the NanoLab at ARROW@TU Dublin. It has been accepted for inclusion in Articles by an authorized administrator of ARROW@TU Dublin. For more information, please contact arrow.admin@tudublin.ie, aisling.coyne@tudublin.ie, gerard.connolly@tudublin.ie. Funder: the Science Foundation Ireland Research Frontiers Program PHY037 2006. The Raman Instrument was purchased under the framework of the INSPIRE programme, funded by the Irish Government's Programme for Research in Third Level Institutions, Cycle 4, National Development Plan 2007-2013, supported by the European Union Structural Fund. 
Authors

Qiaohuan Cheng, Sourabhi Debnath, Elizabeth Gregan, and Hugh Byrne

This article is available at ARROW@TU Dublin: https://arrow.tudublin.ie/nanolart/34 


\title{
Ultrasound-assisted SWNTs dispersion: effects of sonication parameters and solvent properties
}

\author{
Qiaohuan Cheng*, Sourabhi Debnath, Elizabeth Gregan and Hugh J. Byrne
}

FOCAS Research Institute/School of Physics, Dublin Institute of Technology, Kevin Street, Dublin 8, Ireland

*Corresponding author. E-mail: qiaohuan.cheng@ dit.ie. Tel: +353 14027931.

\section{ABSTRACT}

Ultra-sonication is widely used for preparing Single-Walled Carbon Nanotube (SWNT) dispersions in different solvent media and it has been shown to play a critical role in dispersing and debundling SWNTs. The strong shear force which can exfoliate the SWNT bundles during sonication comes from cavitation, which entails a process of bubble formation, growth and collapse. The efficiency of the cavitation process is closely correlated to many solvent parameters, including vapour pressure, viscosity, surface tension, as well as the sonication frequency, intensity and time. In this study, SWNTs were dispersed in a range of organic solvents assisted by tip sonication. The effects of sonication intensity and time were investigated in ortho-dichlorobenzene (o-DCB) and dimethylformamide (DMF). The aggregation fraction below the dispersion limit of SWNTs in the range of organic solvents was found to be influenced by the solvent parameters, particularly solvent vapour pressure and viscosity. It is demonstrated that the parameters associated with the sonication process rather than solvent solubility 
parameters govern the dispersion process. It is further confirmed that significant degradation of the SWNTs is affected during the dispersion process.

KEYWORDS: SWNTs dispersion, sonication, solvents, vapor pressure, viscosity

\section{INTRODUCTION}

Due to strong inter tube van der Waals forces ${ }^{1}$, Single-Walled Carbon Nanotubes (SWNTs) are produced in bundles and are not readily soluble in either water or common organic solvents. Good dispersion has been achieved using surfactants ${ }^{2}$ or biomolecules ${ }^{3}$ in water, and organic polymers and molecules in non-solvents ${ }^{4}$. A range of organic solvents have been explored as dispersion media, with varying degrees of success ${ }^{5-9}$. Attempts to identify the optimal solvent properties have been based on solubility parameters such as Hildebrandt or Hansen parameters ${ }^{8-13}$ or surface energy ${ }^{14}$. However, little agreement between the different studies is apparent.

In a systematic study of chlorinated aromatic solvents ortho-dichlorobenzene (o-DCB), monochlorobenzene (MCB), meta-dichlorobenzene ( $m$-DCB) and 1, 2, 4-trichlorobenzene (TCB)) and other solvents reported in literature, including dimethylformamide (DMF) ${ }^{5,7}$, 1,2-dichloroehtane (DCE) ${ }^{15}$, chloroform ${ }^{16}$, and toluene ${ }^{17}$, it was found that SWNTs are easily dispersed in solvents with Hildebrand solubility parameter range from $\sim 22-24 \mathrm{MPa}^{1 / 2}$ and Hansen polarity component $\left(\delta_{\mathrm{P}}\right) \sim 12-14$ $\mathrm{MPa}^{1 / 2}$. No clear correlation between dispersion limits and the dispersion force $\left(\delta_{\mathrm{D}}\right)$ or hydrogen bonding force $\left(\delta_{\mathrm{H}}\right)$ were evident ${ }^{13}$. Bergin et al. identified optimal solubilisation for solvent Hildebrand parameters in the range $19<\delta<24 \mathrm{MPa}^{1 / 2}$, with a maximum at $21 \mathrm{MPa}^{1 / 2}$. The corresponding optimal ranges for the Hansen parameters were, $\delta_{\mathrm{D}}: 17<\delta_{\mathrm{D}}<19 \mathrm{MPa}^{1 / 2}, \delta_{\mathrm{P}}: 5<\delta_{\mathrm{P}}<14 \mathrm{MPa}^{1 / 2}, \delta_{\mathrm{H}}: 3<$ $\delta_{\mathrm{H}}<11 \mathrm{MPa}^{1 / 2}$, and the estimated parameters for optimum solubilization of SWNTs were $\delta_{\mathrm{D}}=17.8$ 
$\mathrm{MPa}^{1 / 2}, \delta_{\mathrm{P}}=7.5 \mathrm{MPa}^{1 / 2}, \delta_{\mathrm{H}}=7.6 \mathrm{MPa}^{1 / 29}$. In the study of Detriche et al. of CVD SWNTs, the optimal

Hildebrand range was found to be $20<\delta<22 \mathrm{MPa}^{1 / 2}$, with Hansen parameters $\delta_{\mathrm{D}}: 19<\delta_{\mathrm{D}}<21$ $\mathrm{MPa}^{1 / 2}, \delta_{\mathrm{P}}: 4<\delta_{\mathrm{P}}<7 \mathrm{MPa}^{1 / 2}, \delta_{\mathrm{H}}: 3<\delta_{\mathrm{H}}<5 \mathrm{MPa}^{1 / 2}$, and the estimated parameters for optimum solubilization of SWNTs were: $\delta_{\mathrm{D}}=19.4 \mathrm{MPa}^{1 / 2}, \delta_{\mathrm{P}}=6.0 \mathrm{MPa}^{1 / 2}, \delta_{\mathrm{H}}=4.5 \mathrm{MPa}^{1 / 2}$. A further study by Ham et al. identified $\delta_{\mathrm{D}}$ as the most important parameter with values in the range $17<\delta_{\mathrm{D}}<18 \mathrm{MPa}^{1 / 2}$, $\delta_{\mathrm{P}}$ as having an upper limit of $14 \mathrm{MPa}^{1 / 2}$, and $\delta_{\mathrm{H}}$ an upper limit of $12 \mathrm{MPa}^{1 / 2}$.

There is thus considerable discrepancy between the studies already reported in literature and it is notable that the types of nanotubes and the preparation conditions vary significantly between studies. Notably, ultrasonication is universally employed to assist the dispersion and stabilization of SWNTs ${ }^{5-}$ $7,9,13,14,16$. However, there is no standard procedure for the sonication process, different groups applying different sonication treatment to their samples. Table 1 summarizes some of the sonication parameters which have been used for dispersing and stabilizing SWNTs in liquids. It is clearly seen that the sonication conditions vary significantly, including sonicator types, sonication times and temperature control. For example, the sonication times vary from 2 mins to 30 mins for tip sonication and 30 mins to $20 \mathrm{hrs}$ for bath sonication respectively.

The strong shear force which can exfoliate the SWNT bundles during sonication comes from the cavitation process, which entails bubble formation, growth and collapse. This process is intimately dependant on many factors ${ }^{18}$, including: (a) The nature of the solvent, notably the solvent viscosity, surface tension, vapour pressure, gas solubility and type of active intermediates or radicals formed, (b) The nature of gas solubilised in the liquid which can change the number of cavitation events and gas content. (c) Ambient liquid temperature and pressure. As many of the solvent parameters are temperature dependent, a change of temperature will effect the liquid properties and the gas solubility. (d) Applied intensity. The intensity of ultrasound influences the size of cavitation and therefore the probability of cavitation events per unit volume. The larger the intensity the larger will be the acoustic 
amplitude and collapse pressure and hence the faster and more violent the collapse. (e) Ultrasound frequency. While the acoustic frequency is increased, the size of the cavitation bubble decreases, resulting the influence of the cavitation threshold. An increase in frequency means shorter acoustic periods, lower maximum bubble size, and thus less cavitation intensity. (f) The sonication time which determines the total energy input. Many of these parameters are correlated, as for instance, most of the solvent parameters are temperature dependent, therefore, increasing the complexity of the study.

To further complicate the issue, it has been reported that strong sonication can not only exfoliate the SWNT bundles but also induce defects and even scission of the tubes ${ }^{19}$. The damage of the SWNTs is normally monitored by Raman spectroscopy, an increase of the intensity of the defect or D band compared to the corresponding graphitic or $\mathrm{G}$ band intensity being considered to be a measure of damage to the tubes ${ }^{6,20}$.

In order to demonstrate the critical effect of sonication on the debundling and dispersion of SWNTs in organic solvents, and to investigate the damage caused by different sonication treatment, a systematic study of the dispersion of as-produced HiPco SWNTs in a series of organic solvents has been conducted. By measurement of the UV-visible-NIR absorbance before and after centrifugation as a function of concentration, the dispersion limit $\left(\mathrm{D}_{\mathrm{L}}\right)$ of SWNT in each solvent ${ }^{6}$, defined as the concentration at which aggregates cease to dominate the dispersion, as well as the aggregation fraction $\left(\chi_{\text {agg }}\right)$ below the dispersion limit was obtained, as described by Equation 1.

$$
\chi_{\text {agg }}=\frac{A_{\text {before }}-A_{\text {after }}}{A_{\text {before }}}
$$

Equation 1

where $A_{\text {before }}$ is the absorbance (chosen at $660 \mathrm{~nm}$ ) before centrifugation and $A_{\text {after }}$ is the absorbance at the same wavelength after centrifugation. The debundling and dispersion process, as characterised by the dispersion limit and the aggregation fraction, is seen to correlate well with the solvent parameters associated with sonication suggesting that these rather than the solubility parameters govern the 
dispersion process. Raman spectroscopy of $o$-DCB and DMF dispersions demonstrates significant damage to the SWNTs which is well correlated with the increased solubility suggesting that the use of universal solubility parameters are not appropriate.

\section{EXPERIMENTAL}

HiPco SWNTs from Carbon Nanotechnologies Inc., batch number PO341, were used as received.

All the sonication treatments in this study were carried out by an Ultrasonic processor VCX $750 \mathrm{~W}$ (SONICS \& MATERIALS, INC.), of frequency $20 \mathrm{kHz}$ with the output power set at $26 \%$.

$4.2 \mathrm{mg}$ SWNTs were added into $20 \mathrm{ml}$ solvent. The initial dispersion was produced by sonicating for 20s, whereupon it was serially diluted by 0.75 to produce a range of dispersions with concentrations from $0.21 \mathrm{mg} / \mathrm{ml}$ to $0.001 \mathrm{mg} / \mathrm{ml}$. The volume of each sample was $5 \mathrm{ml}$. All samples were then sonicated for an additional $100 \mathrm{~s}^{21}$ to make sure each sample received the same sonication treatment. All the dispersions were subsequently centrifuged at 3000rpm ( $945 \mathrm{~g})$ (ECONOSPIN Sorvall Instruments) for 60 minutes. An identical procedure was performed in all the employed solvents.

In order to investigate the effect of sonication time and output power of the sonicator, dispersions in two solvents, $o$-DCB and DMF, were chosen. $3.2 \mathrm{mg}$ SWNTs were added to $80 \mathrm{ml} o$-DCB and DMF respectively $(0.04 \mathrm{mg} / \mathrm{ml})$. This initial dispersion was sonicated for $20 \mathrm{~s}$ with the output power of the sonicator set as 26\%. The dispersion was then immediately divided into 16 bottles, each sample containing $5 \mathrm{ml}$. 6 samples of each solvent were chosen for the study of output power of the sonicator. The output power of the sonicator was varied between $21 \%$ and $38 \%$. The remaining 10 samples were used to investigate the effect of sonication time from $20 \mathrm{~s}$ to $220 \mathrm{~s}$, in 20 s intervals for each sample. All 
the samples were allowed to settle for 2 days before a mild centrifugation was carried out to remove large aggregates.

UV-vis-NIR absorption (Perkin-Elmer Lambda 900) measurements were performed both before and after centrifugation. Measurements were performed using an integrating sphere to minimize the contributions of scattering to the measured extinction ${ }^{8,22} \cdot 10$-millimeter quartz cuvettes were used for all the measurements. UV-vis-NIR measurements were carried out on the whole sample before centrifugation (immediately before measurement all samples were vigorously shaken) but only on the supernatant after centrifugation, so that the mass fraction of aggregates can be estimated (see Equation 1). The absorbance at a wavelength of $660 \mathrm{~nm}$ was used for all the calculations ${ }^{6,8,21}$.

Raman measurements were performed with a LabRAM HR800 Raman Microscope (Horiba Jobin Yvon) at laser energy $2.33 \mathrm{eV}(532 \mathrm{~nm})$ on the supernatant of each sample (after centrifuge) drop cast onto glass substrates. A $\times 50$ objective lens was used for all the measurements. The spot size and laser power at the sample were approximately $2 \mu \mathrm{m}$ and $30 \mathrm{~mW}$ respectively. Up to ten spectra were taken randomly for each sample. The intensities of the $\mathrm{D}$ and $\mathrm{G}^{+}$bands were taken after base line correction and the ratios of $\mathrm{I}_{\mathrm{D}} / \mathrm{I}_{\mathrm{G}}{ }^{+}$were calculated for all spectra and averaged.

\section{RESULTS AND DISCUSSION}

Figure 1 shows the concentration dependence of the calculated aggregation fraction for SWNTs in MCB, $o$-DCB, TCB and DMF dispersions. In the example of TCB, the aggregation fraction decreases with decreasing concentration until it reaches a minimum of $\sim 0.1$ below the dispersion limit. A complete study of the variation of dispersion limit with solvent has recently been reported ${ }^{13}$. AFM studies have confirmed that the reduction of the aggregation fraction is due to exfoliation of the bundles with decreasing concentration until the bundles are maximally dispersed. For the range of solvents, in 
addition to the variation of the dispersion limit, the aggregation fractions below $\mathrm{D}_{\mathrm{L}}$ also vary significantly for the different solvents, but the two are not correlated. For example, the dispersion limit of SWNTs in DMF can be considered to be $\sim 0.022 \mathrm{mg} / \mathrm{ml}$, and the aggregation fraction below $\mathrm{D}_{\mathrm{L}}$ is around 0.5. However, the dispersion limit of SWNTs in TCB is only $\sim 0.005 \mathrm{mg} / \mathrm{ml}$, but the aggregation fraction below $\mathrm{D}_{\mathrm{L}}$ is found to be 0.1 . AFM has demonstrated that a high aggregation fraction is due to only partial debundling of SWNTs aggregates during the sonication ${ }^{13}$. The dispersion limit and aggregation fraction of SWNTs below $\mathrm{D}_{\mathrm{L}}$ in each solvent are listed in Table 2, together with some of the physical parameters of the solvents, including molecular weight, viscosity, vapour pressure, density and surface tension.

It is surprisingly to find that the aggregation fraction below the dispersion limit in each solvent varies significantly and that there is no correlation with the dispersion limit of SWNTs in the corresponding solvent. As all the samples received the same sonication treatment, this variation is attributed to the difference of solvent properties during sonication. In order to further explore this, a further two sets of SWNTs/DMF dispersions were sonicated for 4 mins and 6 mins respectively. The aggregation fractions for different sonication times were plotted as a function of prepared concentration, shown in Figure 2, and compared to those presented in Figure 1. It is clear that the degree of debundling, or aggregation fraction, below the dispersion limit is critically dependent on sonication time. The estimated dispersion limit, reflecting the efficiency of debundling, appears to be largely unaffected by the degree of sonication, however, indicating that it may be determined by the solvent parameters rather than the sonication conditions.

In order to further investigate the effect of sonication time on the dispersion of SWNTs, a series of $0.04 \mathrm{mg} / \mathrm{ml} \mathrm{SWNTs} \mathrm{in} o$-DCB and DMF dispersions were made with sonication times varying from 20 $\mathrm{s}$ to $220 \mathrm{~s}$. The absorbance of each sample after centrifugation was measured by UV-vis-NIR spectroscopy and plotted as a function of the sonication time (t), shown in Figure 3. It is clearly seen 
that increased sonication time increases the dispersion of SWNTs in both solvents. In $o$-DCB, the absorbance appears to reach a plateau in the region 120-160 sec, whereas in DMF the absorbance continues to increase upon sonication up to $\sim 200 \mathrm{sec}$.

Figure 4 shows the absorbance of the SWNTs dispersions in $o$-DCB and DMF at $0.04 \mathrm{mg} / \mathrm{ml}$ as a function of sonication power for a fixed time of $120 \mathrm{sec}$. The absorbance of $o$-DCB solution reaches a maximum for an output power of $26 \%$. However, for the DMF solution, the optimum output power was found to be $30 \%$.

The sonication conditions of $120 \mathrm{sec}$ and $26 \%$ output power were established in a previous study for $o$-DCB, wherein it was observed that the absorbance before centrifugation was seen to be maximised. Clearly this is not quite the case for the dispersions after sonication, but most importantly, the optimal sonication conditions are solvent dependent.

According to the theory of ultrasonic processes, the cavitation effect is strongly dependent on solvent parameters, notably the solvent vapour pressure, viscosity and surface tension ${ }^{18}$. The aggregation fractions of the dispersions of SWNTs in different solvents, sonicated for 2 mins at $26 \%$ output power, were plotted as a function of the solvent vapour pressures, shown in Figure 5 (a). There is clearly a correlation between the aggregation fraction of SWNTs in each solvent and the solvent vapour pressure although a number of "outliers" are apparent. The aggregation fraction drops significantly when the vapour pressure of the solvent is below $10 \mathrm{~mm} / \mathrm{Hg}$, indicating that sonication in solvents with lower vapour pressure more effectively debundle and disperse the SWNTs aggregates. In low vapour pressure solvents, more energy is require to induce cavitation, and consequently more energy is released upon bubble collapse. This energy is then available to aid in the dispersion of SWNTs ${ }^{18}$. 
In sonochemistry, the variation of the decomposition rate of, for example, $\mathrm{Fe}(\mathrm{CO})_{5}$ in organic solvents is strongly dependent on the vapour pressure, a linear correlation between $\ln (k)$, where $k$ is the decomposition rate constant and solvent vapour pressure being observed ${ }^{23}$. If the debundling process of nanotube aggregates is comparable to the decomposition of $\mathrm{Fe}(\mathrm{CO})_{5}$, a similar correlation should be observed. The dashed line of Figure 5 is a fit of an exponential dependence of $\left(1-\chi_{\text {agg }}\right)$, representing the debundling rate, on the solvent vapour pressure. Excluding the "outliers", an excellent fit is observed indicating that the debundling process can be modelled according to the principles of sonochemistry.

Figure 6 shows the aggregation fraction below the dispersion limit in each solvent as a function of solvent viscosity. Again a good correlation is observed, again with some "outliers". Notably, the outliers are also outliers in Figure 5. Lower aggregation fraction and therefore better dispersion is observed in higher viscosity solvents. Although viscous solvents are known to increase the threshold of the cavitation ${ }^{24}$, the effects resulting from cavitation collapse in viscous liquids are stronger than collapse in less viscous liquid, resulting in more efficient debundling of $\mathrm{SWNTs}^{24}$.

Figure 7 shows the aggregation fraction as a function of solvent surface tension. If DMSO, DCE, and chloroform are again assumed to be anomalous, then a reasonable correlation between the aggregation fraction and the solvent surface tension may be inferred. As is the case for viscosity, the initiation of the cavitation process requires more energy in viscous solvents and therefore more energy is released upon collapse, resulting in more efficient dispersion of the SWNT aggregates.

Over the range of solvents, therefore, a good correlation of the solvent parameters governing the sonication process is observed, indicating that this process, rather than conventional solubilisation, is predominant in the dispersion of nanotubes. Notably, it might be expected that parameters such as those described by Hildebrant or Hansen might be more relevant for in the regime of high dispersion (i.e. low 
aggregation fraction). However, the correlation with the sonication parameters appears to extend to this region.

Given the many factors involved in the sonication process, it is not surprising that there is a significant spread observed in all the plots. It is noted, however, that certain solvents are consistently observed as outliers. DMSO is known to readily absorb water from the environment ${ }^{25}$, which might be the reason for DMSO behaves anomalously. The deviation of the aggregation fractions in Chloroform and DCE is probably due to the degradation of the solvents themselves and the formation of $\mathrm{Cl}_{2}$ and $\mathrm{HCl}$ during sonication ${ }^{26}$. It is notable however chlorinated aromatic solvents, reported to polymerise under sonication $^{26,27}$, do not appear as outliers. The outlying behaviour of DBE might similarly be due to solvent degradation or alternatively to the extremely high density of DBE compare to other solvents.

For all solvents, parameters such as viscosity are intimately related to the solvent density and thus it is reasonable to expect that the efficiency of the sonication process can be correlated to the solvent density. This is indeed the case, as shown in Figure 8. Furthermore, if a constant value for the molecular volume can be simplistically assumed, one would therefore expect a correlation between solvent molecular weight and dispersion efficiency. This is indeed the case as shown in Figure 9, suggesting a relatively simple solution to the optimisation of the solubilisation process.

However, ultrasonication not only affects the exfoliation of the SWNTs bundles, it also induces defects and even scission of the tubes ${ }^{19,28}$. The process is commonly monitored via the ratio of the D and $G$ bands of the Raman spectrum, $I_{D} / I_{G}{ }^{6,20}$. It has been demonstrated that changes in the $I_{D} / I_{G}$ ratio as a result of sonication are predominately due to nanotube scission ${ }^{28}$, and that the ratio scales inversely with the average nanotube length. 
Figure 10 plots the $\mathrm{I}_{\mathrm{D}} / \mathrm{I}_{\mathrm{G}}$ ratio in drop cast deposits of $o$-DCB and DMF dispersions sonicated for varying times. Clearly there is a significant change in the ratio, and therefore the average nanotube length, as a result of sonication. It has also been shown that sonication-induced cutting results in the

mean tube length decaying as $t^{-1 / 2} 19$. This suggests that $I_{D} / I_{G}$ should increase as $ل_{t}$, as indicated by the dashed lines of the plot. In both cases, the degradation rate is highest over the first $60-80$ seconds, whereupon it approaches a plateau. In $o-\mathrm{DCB}$, the ratio has increased by a factor of 1.7 , while in DMF it has increased by 1.4. This implies a reduction of the average SWNT length by factors of 0.6 and 0.7 respectively. A similar behaviour is seen as a function of sonication power, as shown in Figure 11.

Most significantly, for both solvents, as a function of sonication time and power, the variations of the $\mathrm{I}_{\mathrm{D}} / \mathrm{I}_{\mathrm{G}}$ ratios correlate well with the absorbance values of Figures 3 and 4 . The absorbance values after sonication for a fixed period, and therefore the aggregation fraction, are thus correlated with the degree of damage to the nanotubes as a result of sonication. In literature, however, these values are taken as a measure of the solubility of SWNTs in the respective solvents. It is unclear as yet whether the susceptibility of SWNTs to degradation, or the degradation rates, are dependent on nanotube chirality or diameter. To date, however, in terms of variations of $\mathrm{I}_{\mathrm{D}} / \mathrm{I}_{\mathrm{G}}$ ratios as a function of sonication time, the process has been observed in SWNTs produced by HiPco [19] and pulsed laser vaporization [20], suggesting that it is a relatively universal phenomenon.

\section{CONCLUSIONS}

The dispersion and debundling of SWNTs in organic solvents is critically dependent on the sonication process which is closely dependent on many of the physical parameters of the solvent, including vapour pressure, viscosity, surface tension, density and molecular weight. It appears that these parameters, rather than solubility parameters, govern the dispersion process. The dispersion limit, defined as the concentration at which aggregates cease to dominate the (centrifuged) dispersion appears to be largely independent of sonication conditions, whereas the absorbance of the SWNTs dispersion, often used to 
characterise the degree of solubilisation, increases with the sonication time and the output power of the sonicator. It is furthermore clear that sonication results in damage to the nanotubes and choice of solvent should be guided by minimisation of sonication requirements.

\section{ACKNOWLEDGMENT}

This project is funded under the Science Foundation Ireland Research Frontiers Program PHY037 2006. The Raman Instrument was purchased under the framework of the INSPIRE programme, funded by the Irish Government's Programme for Research in Third Level Institutions, Cycle 4, National Development Plan 2007-2013, supported by the European Union Structural Fund.

\section{REFERENCES}

(1) Rao, A. M.; Chen, J.; Richter, E.; Schlecht, U.; Eklund, P. C.; Haddon, R. C.; Venkateswaran, U. D.; Kwon, Y. K.; Tomanek, D. Phys. Rev. Lett. 2001, 86, 3895.

(2) Vaisman, L.; Wagner, H. D.; Marom, G. Adv. Colloid Interface Sci. 2006, 128, 37.

(3) Zheng, M.; Jagota, A.; Semke, E. D.; Diner, B. A.; Mclean, R. S.; Lustig, S. R.; Richardson, R. E.; Tassi, N. G. Nat. Mater. 2003, 2, 338.

(4) Hwang, J. Y.; Nish, A.; Doig, J.; Douven, S.; Chen, C. W.; Chen, L. C.; Nicholas, R. J. J. Am. Chem. Soc. 2008, 130, 3543.

(5) Landi, B. J.; Ruf, H. J.; Worman, J. J.; Raffaelle, R. P. J. Phys. Chem. B 2004, 108, 17089.

(6) Giordani, S.; Bergin, S. D.; Nicolosi, V.; Lebedkin, S.; Kappes, M. M.; Blau, W. J.; Coleman, J. N. J. Phys. Chem. B 2006, 110, 15708. 
(7) Furtado, C. A.; Kim, U. J.; Gutierrez, H. R.; Pan, L.; Dickey, E. C.; Eklund, P. C. J. Am. Chem. Soc.2004, 126, 6095.

(8) Cheng, Q. H.; Debnath, S.; Gregan, E.; Byrne, H. J. J. Phys. Chem. C 2008, 112, 20154.

(9) Bergin, S. D.; Sun, Z. Y.; Rickard, D.; Streich, P. V.; Hamilton, J. P.; Coleman, J. N. Acs Nano 2009, 3, 2340.

(10) Usrey, M. L.; Chaffee, A.; Jeng, E. S.; Strano, M. S. J. Phys. Chem. C 2009, 113, 9532.

(11) Detriche, S.; Zorzini, G.; Colomer, J. F.; Fonseca, A.; Nagy, J. B. J. Nanosci. Nanotechnol. 2008, 8, 6082.

(12) Ham, H. T.; Choi, Y. S.; Chung, I. J. J. Colloid Interface Sci. 2005, 286, 216.

(13) Cheng, Q., Debnath, S., O’Neill, L., Hedderman, T. G., Gregan, E. and Byrne, H. J. J. Phys. Chem. C 2009, J. Phys. Chem. C, 114 (11), 4857-4863 (2010)..

(14) Bergin, S. D.; Nicolosi, V.; Streich, P. V.; Giordani, S.; Sun, Z. Y.; Windle, A. H.; Ryan, P.; Niraj, N. P. P.; Wang, Z. T. T.; Carpenter, L.; Blau, W. J.; Boland, J. J.; Hamilton, J. P.; Coleman, J. N. Adv. Mater. 2008, 20, 1876.

(15) Kim, K. K.; Bae, D. J.; Yang, C. M.; An, K. H.; Lee, J. Y.; Lee, Y. H. J. Nanosci. Nanotechnol. 2005, 5, 1055.

(16) Bahr, J. L.; Mickelson, E. T.; Bronikowski, M. J.; Smalley, R. E.; Tour, J. M. Chem. Commun. 2001, 193.

(17) Hedderman, T. G.; Keogh, S. M.; Chambers, G.; Byrne, H. J. J. Phys. Chem. B 2004, $108,18860$.

(18) Shah, Y. T.; Pandit, A. B.; Moholkar, V. S. 1999.

(19) Hennrich, F.; Krupke, R.; Arnold, K.; Stutz, J. A. R.; Lebedkin, S.; Koch, T.; Schimmel, T.; Kappes, M. M. J. Phys. Chem. B 2007, 111, 1932.

(20) Bergin, S. D.; Sun, Z.; Streich, P.; Hamilton, J.; Coleman, J. N. J. Phys. Chem. C 2010, $114,231$.

(21) Cheng, Q.; Debnath, S.; Gregan, E.; Byrne, H.J. Phys. Stat. Sol. (b). 2008, 245, 1947. 
(22) Priya, B. R.; Byrne, H. J. Journal of Physical Chemistry C 2008, 112, 332.

(23) Mason, T. J.; Lorimer, J. P. ELLIS HORWOOD LIMITED, England 1988.

(24) Mason, T. J.; Lorimer, J. P. Wiley VCH 2002.

(25) Ellson, R.; Stearns, R.; Mutz, M.; Brown, C.; Browning, B.; Harris, D.; Qureshi, S.;

Shieh, J.; Wold, D. Comb. Chem. High Throughput Screening. 2005, 8, 489.

(26) Moonoosawmy, K. R.; Kruse, P. J. Am. Chem. Soc. 2008, 130, 13417.

(27) Niyogi, S.; Hamon, M. A.; Perea, D. E.; Kang, C. B.; Zhao, B.; Pal, S. K.; Wyant, A. E.;

Itkis, M. E.; Haddon, R. C. J. Phys. Chem. B 2003, 107, 8799.

(28) Lucas, A.; Zakri, C.; Maugey, M.; Pasquali, M.; van der Schoot, P.; Poulin, P. J. Phys. Chem. C 2009, 113, 20599.

(29) Detriche, S.; Nagy, J. B.; Mekhalif, Z.; Delhalle, J. J. Nanosci. Nanotechnol. 2009, 9, 6015. 
Table 1 Different sonication conditions for dispersing SWNTs in liquid

\begin{tabular}{|c|c|c|c|}
\hline Sonicator type & Solvent & Sonication time & Reference \\
\hline $\begin{array}{l}\text { Bath \& Tip } \\
\text { Sonicators }\end{array}$ & Water (surfactant) & $\begin{array}{l}1 \min (\text { tip) }+20 \mathrm{~s} \\
\text { (tip) }+3 \mathrm{hrs} \text { (bath) }\end{array}$ & 22 \\
\hline Bath sonicator & $\begin{array}{l}\text { Organic solvents \& } \\
\text { water (surfactant) }\end{array}$ & $20 \mathrm{hrs}$ & 12 \\
\hline Tip Sonicator & Organic solvents & 30mins (ice cooling) & 9 \\
\hline Tip sonicator & Organic solvents & $2 \mathrm{mins}$ & 8 \\
\hline Tip Sonicator & Organic solvents & 2 mins & 29 \\
\hline Bath sonicator & Amide Solvents & $4 \mathrm{hrs}$ & 7 \\
\hline $\begin{array}{l}\text { Bath \& Tip } \\
\text { Sonicators }\end{array}$ & N-methyl-2-pyrrolidone & $\begin{array}{l}2 \operatorname{mins}(\mathrm{tip})+4 \mathrm{hrs} \\
\text { (bath) +1min (tip) }\end{array}$ & 6 \\
\hline Bath sonicator & Alkyl Amide Solvents & $30 \operatorname{mins}\left(40^{\circ} \mathrm{C}\right)$ & 5 \\
\hline Bath sonicator & ortho-dichlorobenzene & $1 \mathrm{hr}$ & 10 \\
\hline
\end{tabular}

Table 2 The dispersion limits of SWNTs and aggregation fraction below $\mathrm{D}_{\mathrm{L}}$ in different solvents together with the solvents physical parameters (all the samples get 2 mins sonication)

\begin{tabular}{ccccccccc}
\hline Name & $\begin{array}{c}\text { Molecular } \\
\text { formula }\end{array}$ & $\begin{array}{c}\text { Dispersion } \\
\text { limit }(\mathrm{mg} / \mathrm{ml})\end{array}$ & $\begin{array}{c}\chi_{\mathrm{agg}} \\
\text { below } \\
\mathrm{D}_{\mathrm{L}}\end{array}$ & $\begin{array}{c}\mathrm{Mw} \\
(\mathrm{g} / \mathrm{mol})\end{array}$ & $\begin{array}{c}\text { Viscosity } \\
(\mathrm{mPa} \cdot \mathrm{s})\end{array}$ & $\begin{array}{c}\text { Vapour } \\
\text { pressure } \\
(\mathrm{mm} / \mathrm{Hg})\end{array}$ & $\begin{array}{c}\text { Surface } \\
\text { tension } \\
(\mathrm{mN} / \mathrm{m})\end{array}$ & $\begin{array}{c}\text { Density } \\
\left(\mathrm{g} / \mathrm{cm}^{3}\right)\end{array}$ \\
\hline $\begin{array}{c}\text { Chloroform } \\
\text { DCE }\end{array}$ & $\mathrm{CHCl}_{3}$ & 0.001 & 0.4 & 119.38 & $0.57^{*}$ & $159^{*}$ & $26.67^{* *}$ & 1.48 \\
$\mathrm{CH} \mathrm{CHCH}_{2} \mathrm{Cl}$ & 0.007 & 0.6 & 98.96 & $0.84^{*}$ & $65.2^{*}$ & $24.07^{* *}$ & 1.253 \\
Toluene & $\mathrm{HCON}_{2}\left(\mathrm{CH}_{3}\right)_{2}$ & 0.022 & 0.5 & 73.09 & $0.92^{*}$ & $2.7^{*}$ & $36.4^{* *}$ & 0.944 \\
\hline
\end{tabular}




\begin{tabular}{ccccccccc}
\hline $\mathrm{MCB}$ & $\mathrm{C}_{6} \mathrm{H}_{5} \mathrm{Cl}$ & $<0.001$ & 0.9 & 112.56 & $0.80^{*}$ & $11.8^{* *}$ & $32.99^{* *}$ & 1.11 \\
$o$-DCB & $\mathrm{C}_{6} \mathrm{H}_{4} \mathrm{Cl}_{2}$ & 0.015 & 0.25 & 147.01 & $1.324^{* *}$ & $1.2^{*}$ & $37^{*}$ & 1.30 \\
$m$-DCB & $\mathrm{C}_{6} \mathrm{H}_{4} \mathrm{Cl}_{2}$ & 0.004 & 0.4 & 147.01 & $1.023^{* * *}$ & $2.145^{* *}$ & $35.43^{* *}$ & 1.288 \\
$\mathrm{TCB}$ & $\mathrm{C}_{6} \mathrm{H}_{3} \mathrm{Cl}_{3}$ & 0.005 & 0.1 & 181.45 & $1.611^{* * *}$ & $0.3^{* *}$ & $-\cdots$ & 1.50 \\
$\begin{array}{c}\text { DBE } \\
\text { Nitromethane }\end{array}$ & $\mathrm{CH}_{2} \mathrm{BrCH}_{2} \mathrm{Br}$ & 0.010 & 0.25 & 187.86 & $1.629^{* * *}$ & $11^{*}$ & $39.55^{* *}$ & 2.17 \\
Acetonitrile & $\mathrm{CH}_{3} \mathrm{NO}_{2}$ & $<0.001$ & 0.9 & 61.04 & $0.61^{* *}$ & $27.8^{*}$ & $36.53^{* *}$ & 1.138 \\
DMSO & $\mathrm{CH}_{3} \mathrm{CN}$ & $<0.001$ & 0.98 & 41.05 & $0.3443^{* *}$ & $73^{*}$ & $28.66^{* *}$ & 0.786 \\
& $\left(\mathrm{CH}_{3}\right)_{2} \mathrm{SO}$ & 0.006 & 0.65 & 78.13 & $1.996^{*}$ & $0.417^{*}$ & $42.92^{* *}$ & 1.1004 \\
\hline
\end{tabular}

$*$ Data at $20{ }^{\circ} \mathrm{C}, * *$ data at $25^{\circ} \mathrm{C}, * * *$ data at $30{ }^{\circ} \mathrm{C}$

\section{FIGURE CAPTIONS}

Figure 1 Fraction of the nanotube aggregates in MCB, $o-\mathrm{DCB}, \mathrm{TCB}$ and DMF dispersions as a function of prepared concentration.

Figure 2 Aggregation fractions of SWNTs in DMF at different sonication time (volume 5ml, without temperature control).

Figure 3 Absorbance of $0.04 \mathrm{mg} / \mathrm{ml}$ SWNTs in $o$-DCB and DMF after centrifugation as a function of sonication time $(\mathrm{t})$ (the vertical line indicates the sonication time applied in previous studies ${ }^{8,21}$ ).

Figure 4 Absorbance of $0.04 \mathrm{mg} / \mathrm{ml} \mathrm{DMF}$ and $o$-DCB dispersions after centrifuge as a function of sonicator output power (the vertical line indicates the output power used in previous studies ${ }^{8,21}$ ).

Figure 5 Aggregation fractions of SWNTs below the dispersion limits in each solvent as a function of the solvent vapour pressure. The dashed line is a fit of an exponential dependence of $\left(1-\chi_{\text {agg }}\right)$ on the solvent vapour pressure.

Figure 6 Aggregation fractions of SWNTs below the dispersion limits in each solvent as a function of the solvent viscosity.

Figure 7 Aggregation fractions of SWNTs below the debundling solvents in each solvent as a function of the solvent surface tension (surface tension data of TCB is missing). 
Figure 8 Aggregation fractions below the dispersion limit in each solvent as a function of the solvents density.

Figure 9 Aggregation fractions below the dispersion limit in each solvent as a function of the solvents molecular weight.

Figure 10 Absorbance and $\mathrm{I}_{\mathrm{D}} / \mathrm{I}_{\mathrm{G}+}$ ratio as a function of sonication time. Filled squares: absorbance of DMF solutions; Filled circles: absorbance of $o$-DCB solutions; Open squares: $\mathrm{I}_{\mathrm{D}} / \mathrm{I}_{\mathrm{G}+}$ ratios for DMF. solutions; Open circles: $\mathrm{I}_{\mathrm{D}} / \mathrm{I}_{\mathrm{G}+}$ ratios for $o$-DCB solutions; the dashed lines indicates a ${ }^{t}$ dependence of the $\mathrm{ID} / \mathrm{IG}^{+}$ratio.

Figure 11 Absorbance and $\mathrm{ID} / \mathrm{IG}^{+}$ratio as a function of sonicator output power.

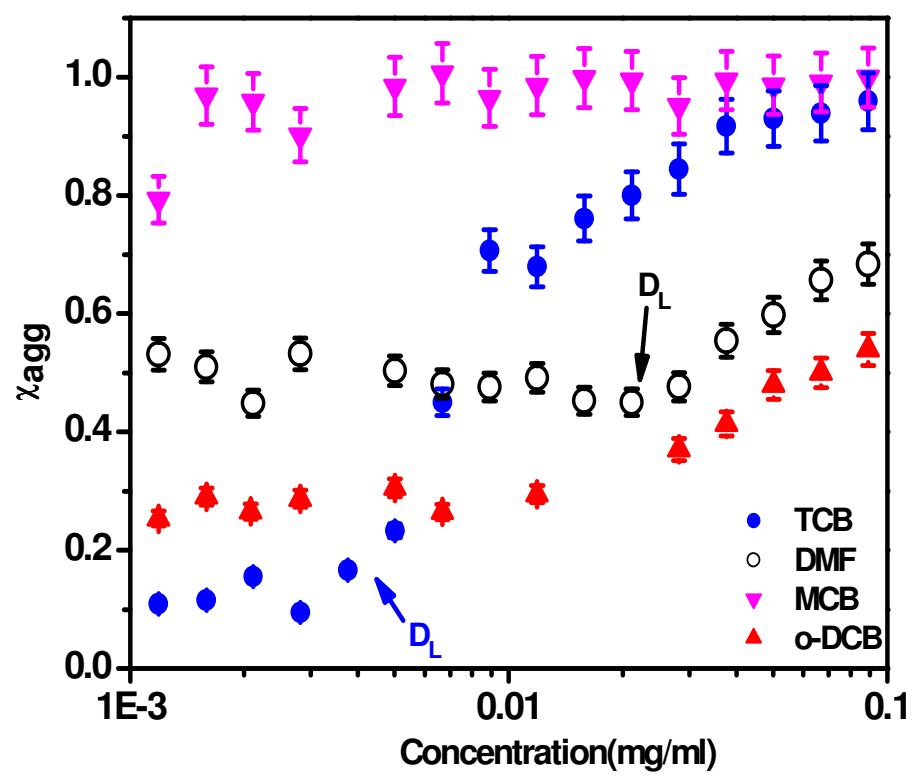

Figure 1 


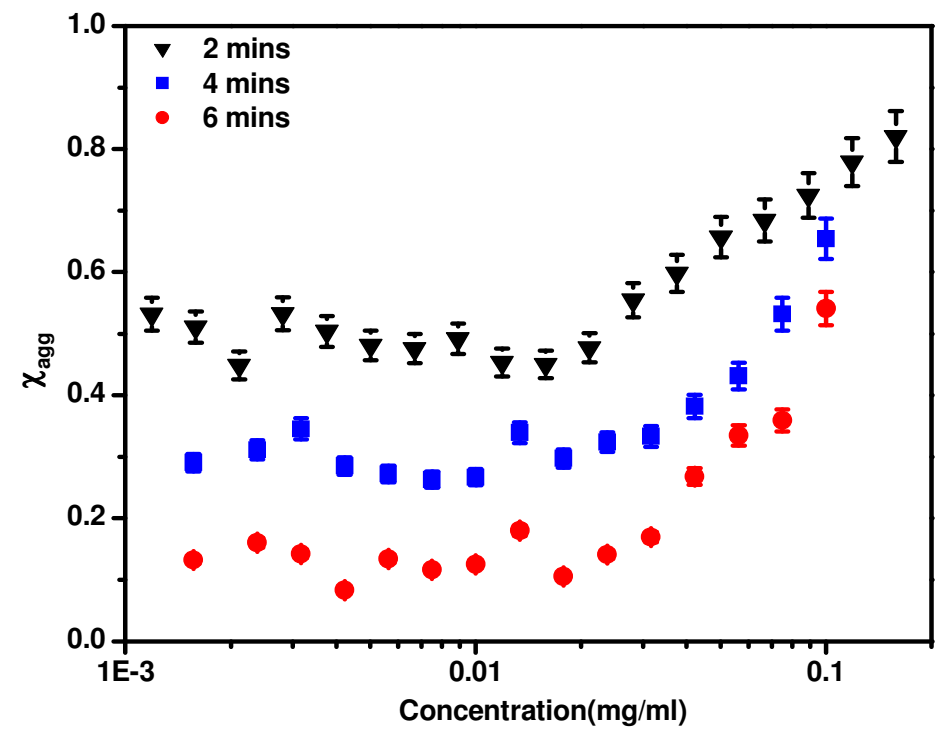

Figure 2

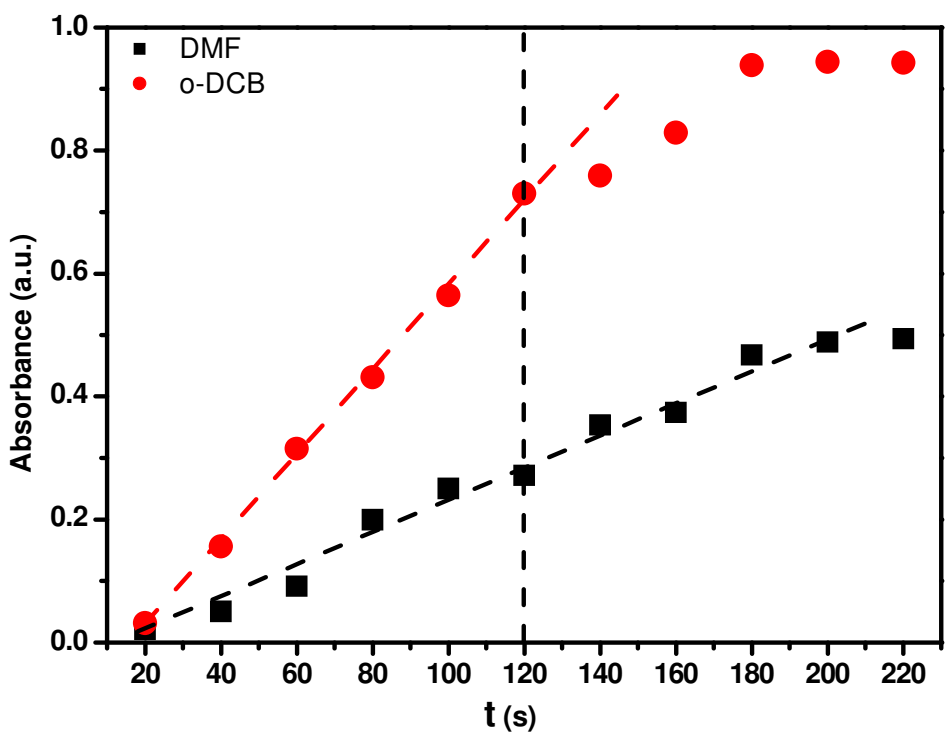

Figure 3 


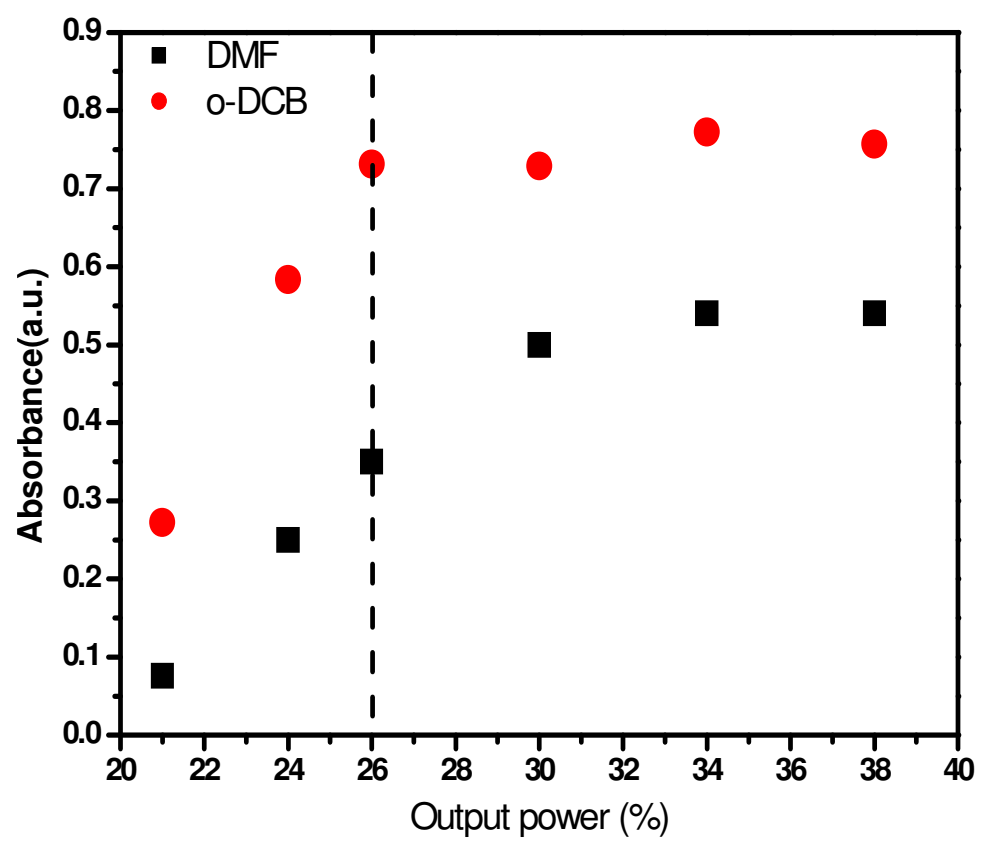

Figure 4

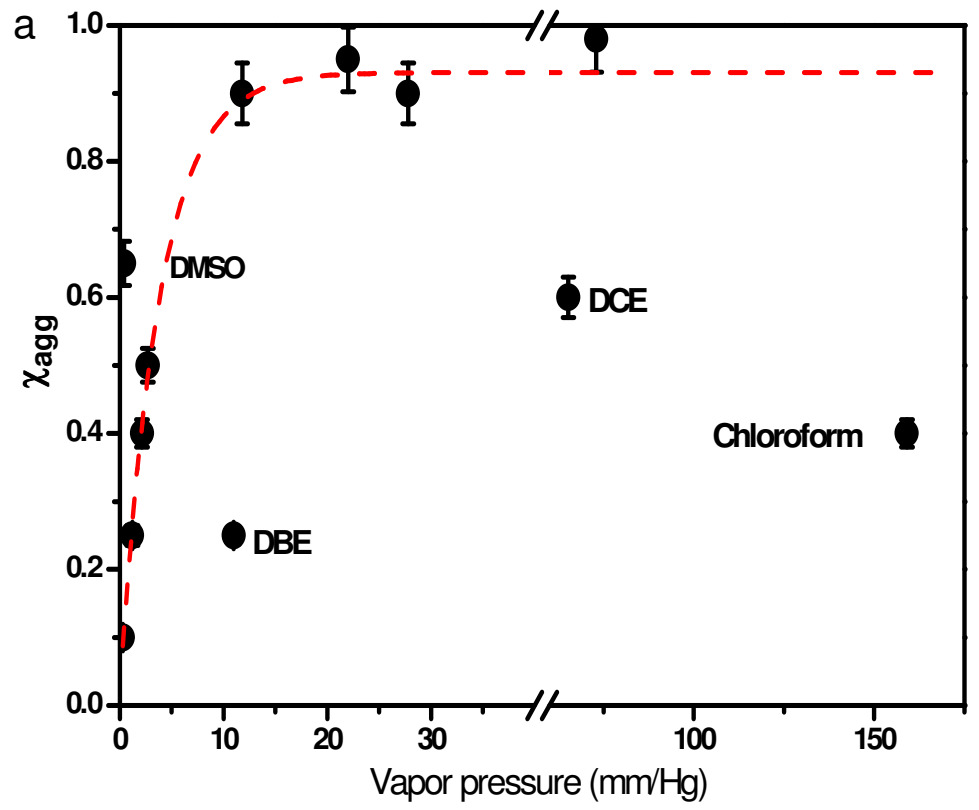

Figure 5 


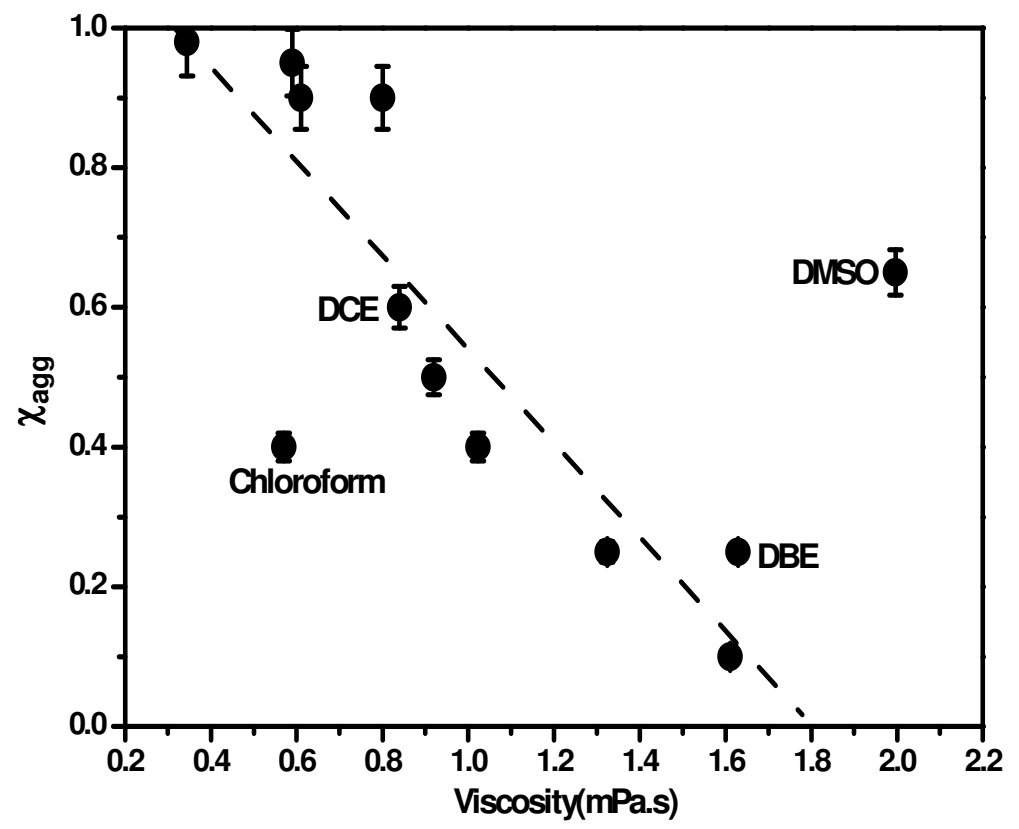

Figure 6



Figure 7 


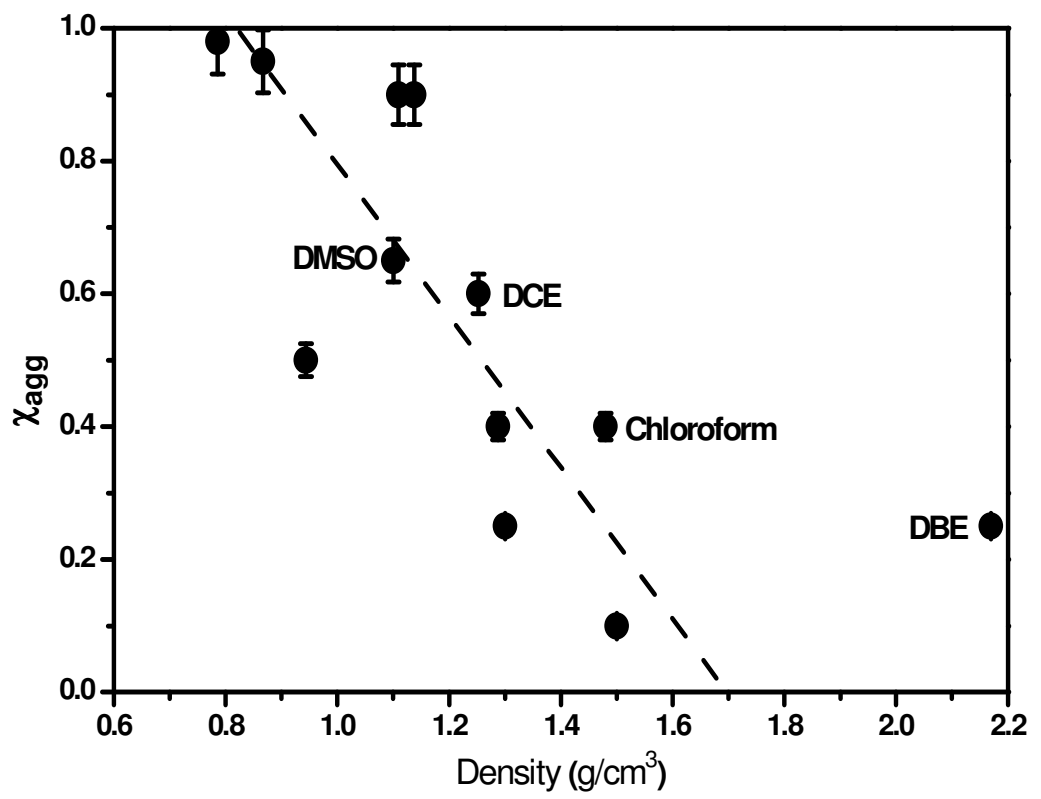

Figure 8

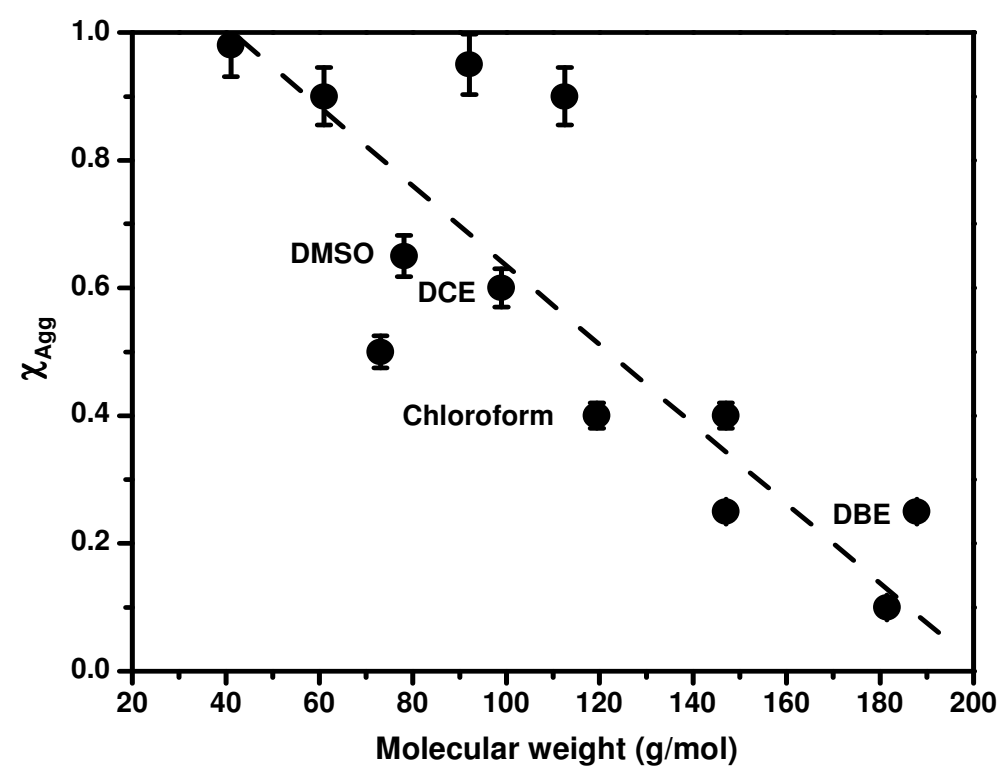

Figure 9 


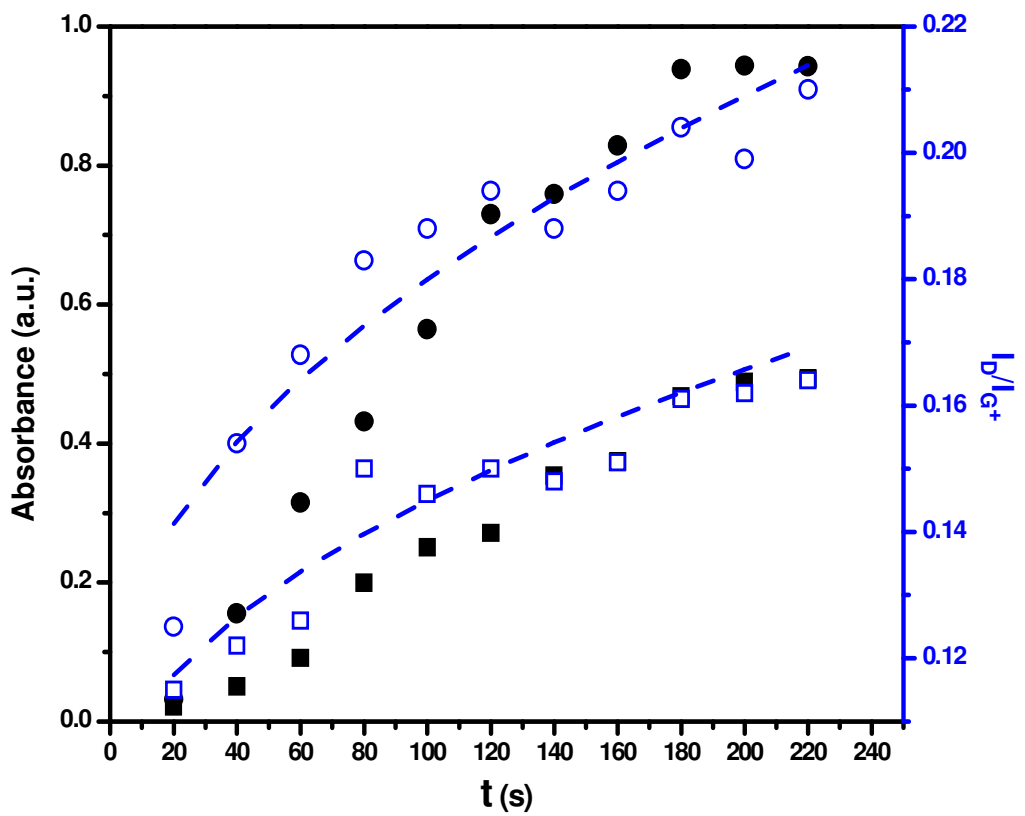

Figure 10

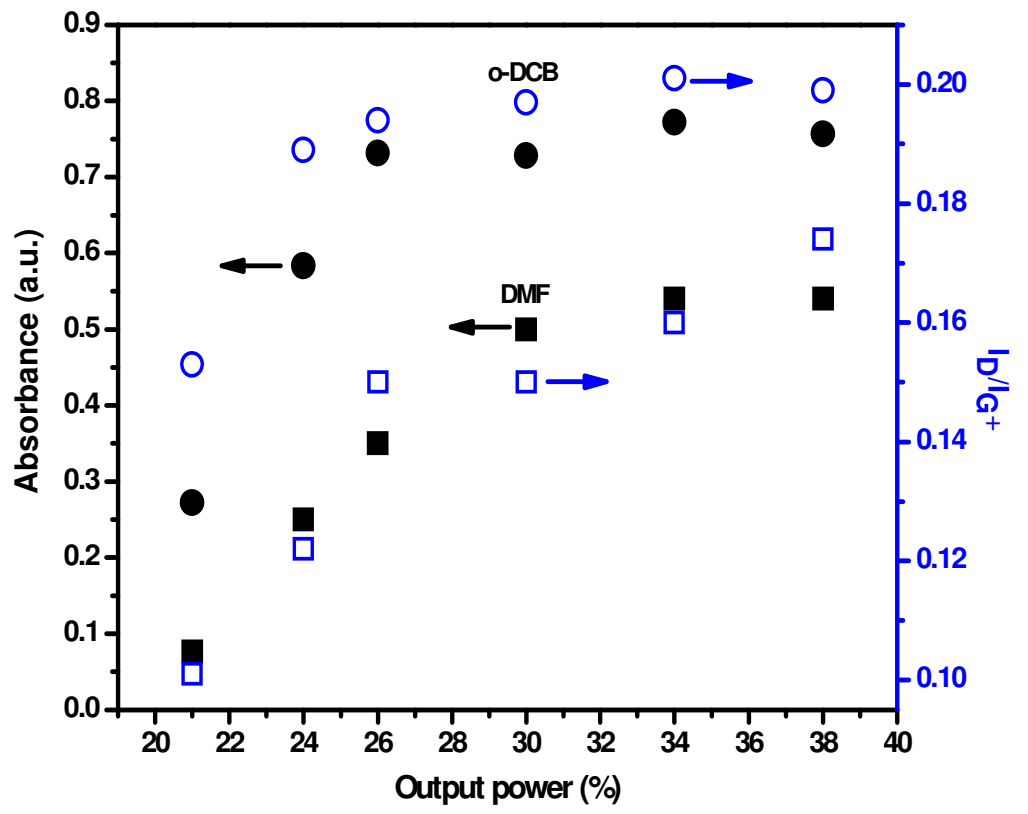

Figure 11 